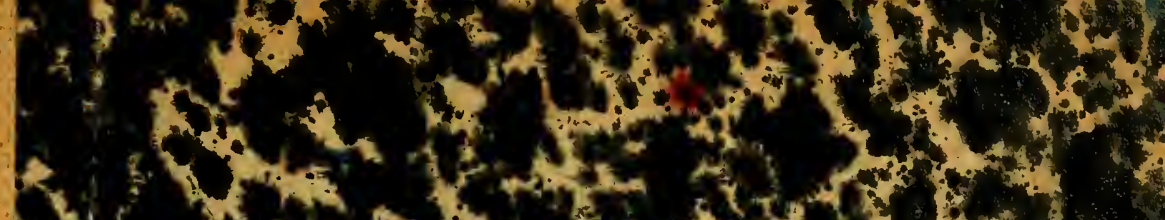

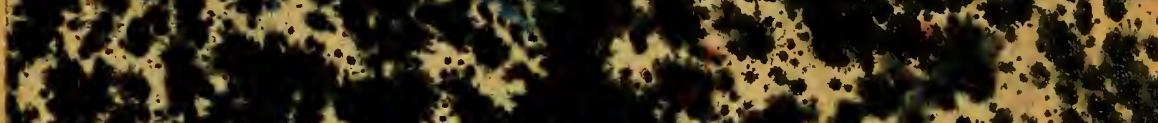

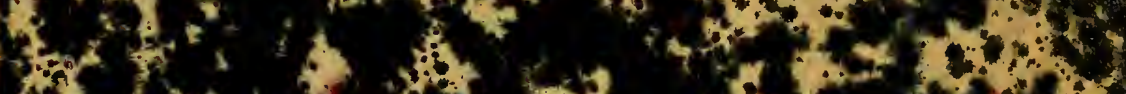

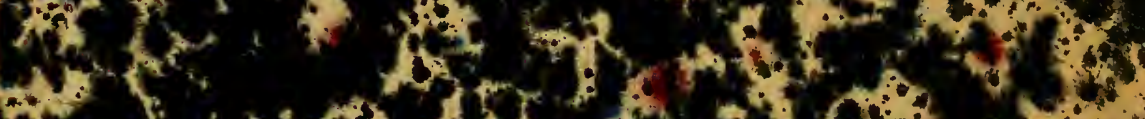

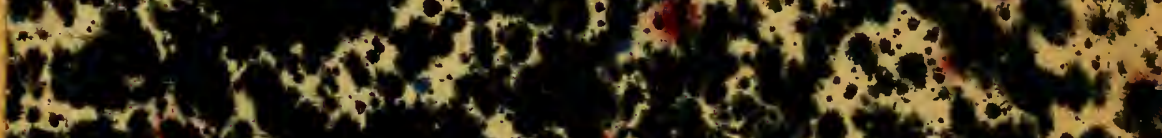

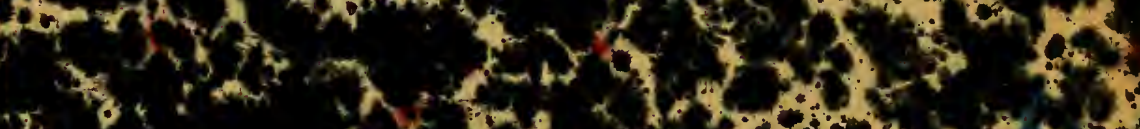

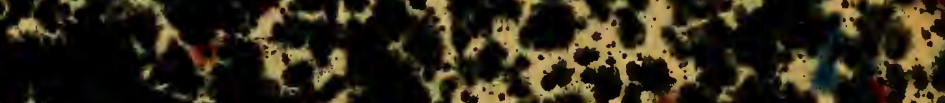

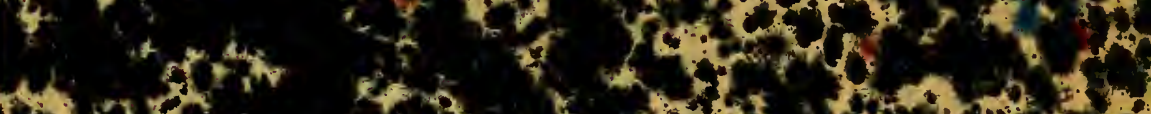

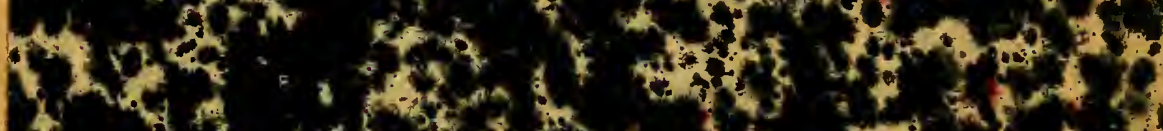

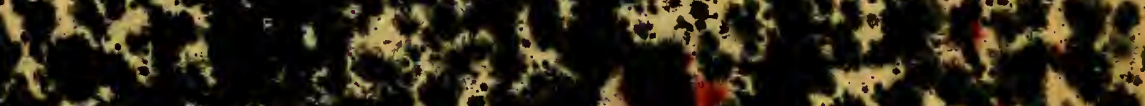

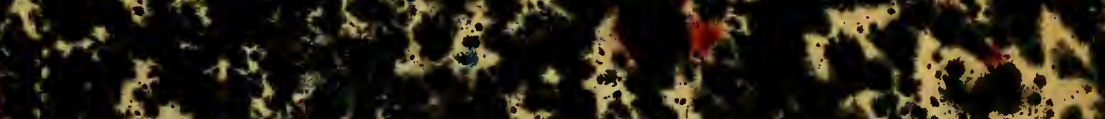

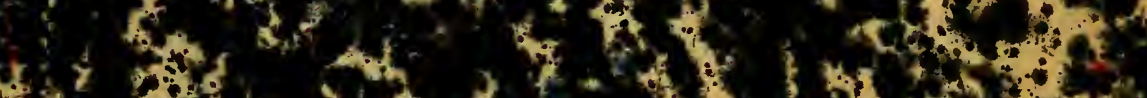

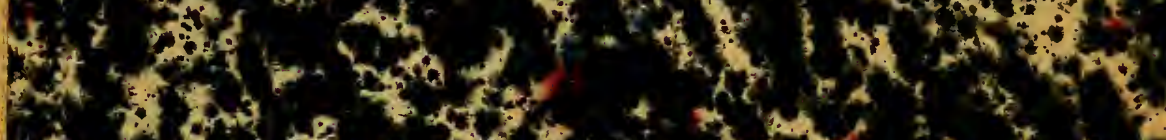

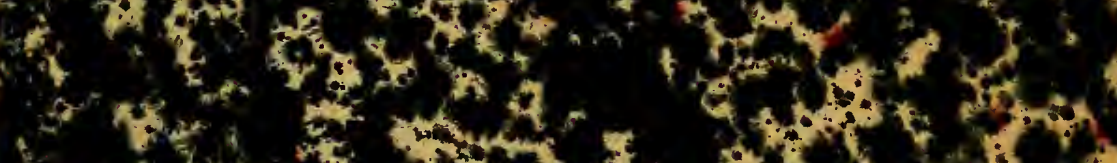

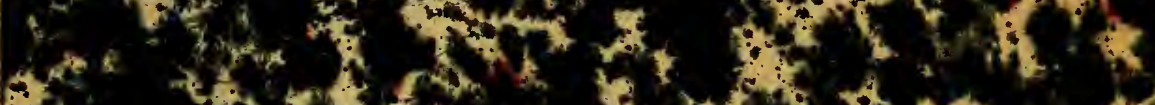

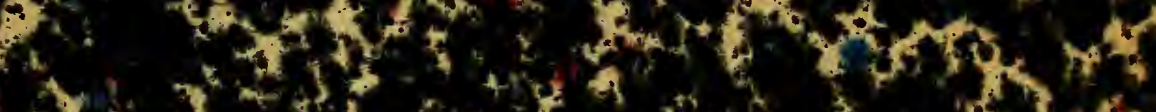

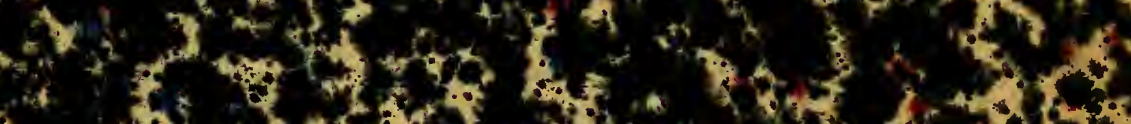
(t)

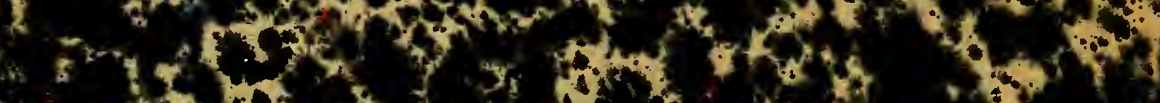

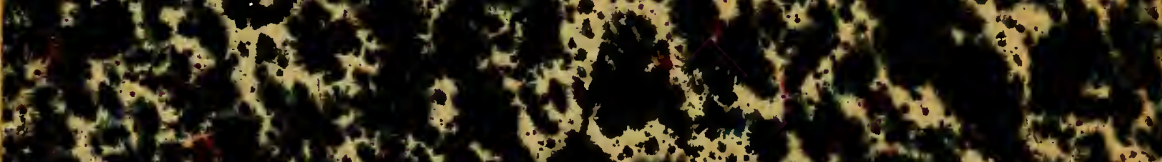

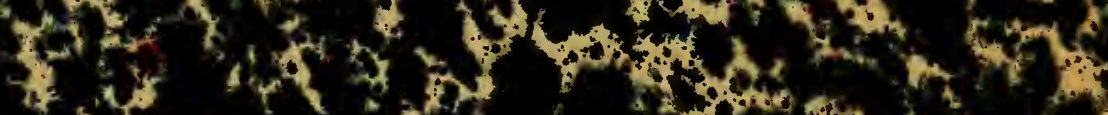

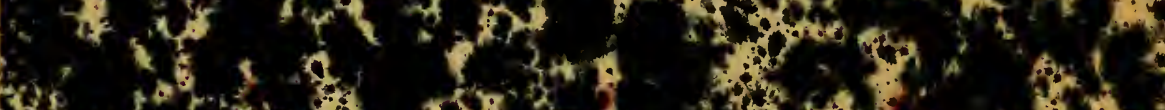

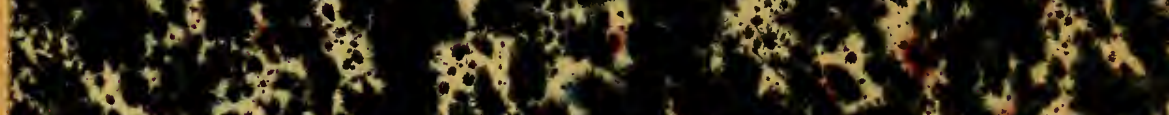

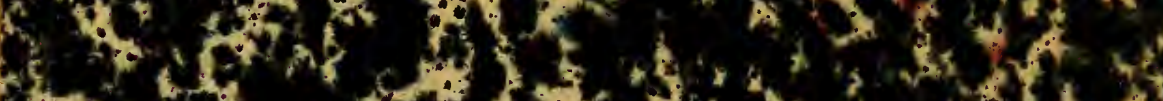
thite

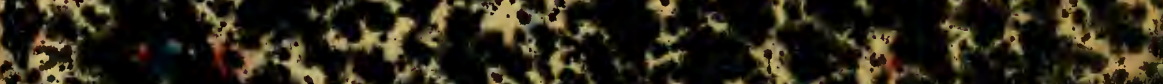

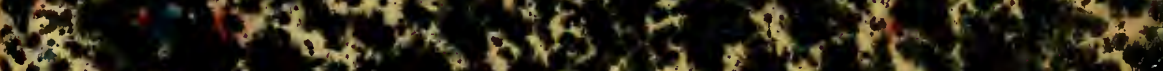

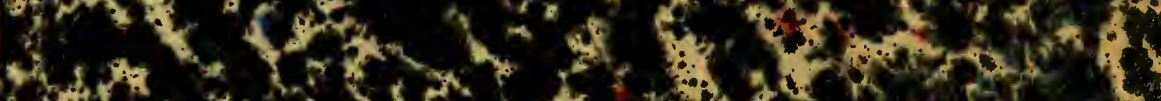

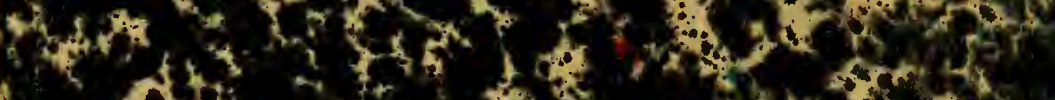

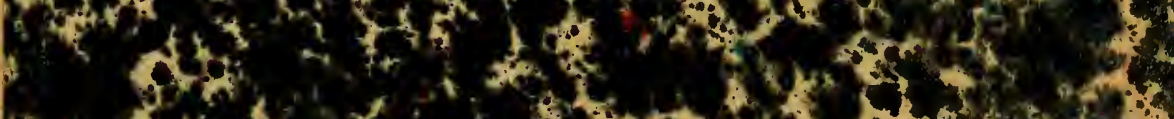

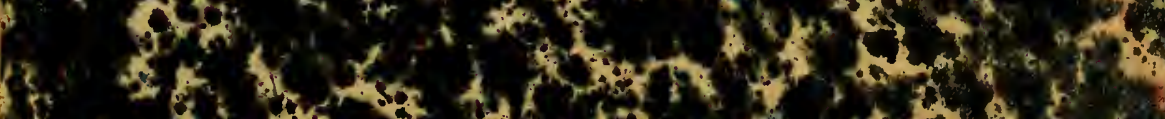

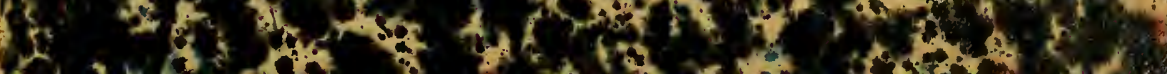




\section{HARVARD UNIVERSITY.}

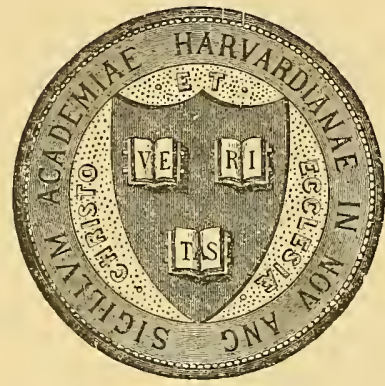

\section{LIBRARY}

OF THE

MUSEUM OF COMPARATIVE ZOÖLOGY.

24,608 .

inchange.

Sovember 17, 1904. 
24.606

(n)

Aus dem pathologischen Institute $\mathrm{zu}$ Kiel.

\section{Ueber Taenienmisbildungen.}

\section{Inaugural-Dissertation}

zur Ex]angung der Doctor'wiirde

der" medicinischen Fakultät

der Kgl. Christian-Albrechts-Universität zu Kiel

$$
\text { vorgelegt von }
$$

Hajo Jelder,

apprub. Arzt

ans Holtland in Ostfriesland.

\section{KIEL,}

Druck von P. Peters

$T_{1900 .}$ 

Aus dem pathologischen Institute zu Kiel.

\title{
Ueber Taenienmisbildungen.
}

\author{
Inaugural-Dissertation \\ zur Frlangung der Doctorwirde \\ der medicinischen Fakultät \\ der Kgl. Christian-Albrechts-Universität zu Kiel

$$
\begin{aligned}
& \text { vorgelegt von } \\
& \text { Hajo Jelden } \\
& \text { approb. Arzt }
\end{aligned}
$$$$
\text { Hajo Jelden, }
$$ \\ aus Holtland in Ostfriesland.
}

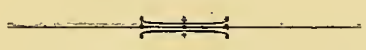

\section{KIEL,}

Druck von P. Peters

1900. 
No. 31.

Rectoratsjahr 1900/1.

Referent Dr. Heller.

Zum Druck genehmigt:

Völckers, z. Zeit Dekan. 
Seinen lieben Eltern 



\section{Bei keiner Art der Darmschmarotzer des Menschen finden}

sich so häufige Abweichungen vom regelmässigen Bau, als bei den Taeniaden, und unter diesen scheint wiederum die Taenia saginata bevorzugt zu sein. Nicht immer sind diese Misbildungen richtig erkannt, und das hat dazu geführt, dass neue Klassen von Bandwürmern aufgestellt wurden. Später hat man jedoch gefunden, dass auch die als Vertreter der besonderen Klassen geschilderten Tiere nur Misbildungen der schon bekannten Taenienarten waren.

Die Misbildungen betreffen die Embryonen von Taenien, die Blasenwürmer und am häufigsten die Bandwürmer selbst.

Bei den Embryonen besteht die häufigste Abnormität in Vermehrung der Haken. Leuliar ${ }^{1}$ ) erwähnt solche Exemplare mit 10-24 Haken und Heller ${ }^{2}$ ) beobachtete Embryonen mit 12, 15. 16 und sogar 32 Haken, die aber nur zum Teil völlig ausgebildet waren. Diese Embryonen sind beträchtlich grösser als im normalen Zustand, zum Teil mchr als doppelt so gross.

Bei Finnen tritt die Misbildung häufig in so hohem Grade auf, dass sie als solche auf den ersten Blick garnicht erkennbar sind. Zenker ${ }^{3}$ ) hat zuerst diese Misbildungen beschrieben und legt ihnen den Namen „traubenförmige Cysticerken-Cysticerkus racemosus" bei, weil die bis $24 \mathrm{~cm}$ langen Schläuche in ihrem Verlaufe zahlreiche mehr oder minder grosse Blasen tragen, die nicht selten wieder gestielt und mit Tochterblasen versehen sind.

1) L., Parasiten des Menschen. 2. Aufl. 1886.

9) Heller \& v. Ziemssen, Handb. der spec. Pathologie u. Therapie VII. 2. Seite 600.

3) Z., Beiträge zur Anatomie und Physiologie, Festgabe für Henle 1882 und Heller, v. Ziemssen, Handbuch der spec. Pathologie u. Therapie, Bd. III., S. 334. 
Während Kü̈chenmeister ${ }^{1}$ ) und Redon ${ }^{2}$ ) die Finne, zu welcher ein Bandwurm niemals gefunden wurde, als Misbildung von Cysticerkus cellulosae ansehen, wollen Leukart ${ }^{3}$ ) und Weinland ${ }^{4}$ ) sie als eine besondere Art - Taenia acanthotrias - betrachtet wissen.

Bei den Echinokokken kommen durch ungleiche Entwickelung der Obertläche buchtige Formen vor. Auch der Echinococcus multilocularis, der faust- bis kopfgrosse Tumoren bildet, lässt sich als besondere Anomalie ansehen.

Misbildungen der Proglottiden sind, besonders bei Taenia saginata, nichts weniger als selten, reduciren sich aber in den meisten Fällen auf eine bald luxuriirende, bald auch unvollständig entwickelte oder gänzlich unterbliebene Segmentirung. Damit hängt auch die Misbildung zusammen, die sich durch Vermehrung der Geschlechtsöffnungen an den einzelnen Gliedern kund thut. Pallas berichtet von Gliedern mit 2 und 3 Geschlechtspapillen, bald an derselben Seite ïbereinander, bald in unregelmässiger Abwechselung an beiden Seiten. Leukart ${ }^{5}$ ) zählte bis zu $5 \mathrm{~Pa}$ pillen an einem Gliede und Collin ${ }^{6}$ ) berichtet von einem $15 \mathrm{~cm}$ langen, ungegliederten Stiicke, das mindestens 25-30 Genitalporen besessen haben muss. Nach Heller ${ }^{7}$ ) kommt auch eine Hächenständige Lage der Geschlechtsöffnungen vor. Man hat sich diese Misbildungen so zu erklären, dass es nicht, wie es auf den ersten Blick vielleicht den Anschein hat, um Glieder handelt, deren Geschlechtsöffnungen vermehrt sind, sondern um Gliederstrecken, in denen die Abtrennung der einzelnen Proglottiden unterblieben ist.

Häufig verläuft die Demarkationslinie derartig, dass ein Glied den Eindruck eines seitlich anhängenden oder überzähligen

1) K., Über die im und am Ienschen vorkommenden Parasiten. 2. Aufl. S. 136.

2) Cpt. rend. 1877. T. 175, p. 676 .

3) L. Parasiten des Menschen, p. 711.

4) $\mathrm{l}, \mathrm{c}$

5) L. Parasiten des Menschen, p. 569.

6) C., Gaz. des hôp. 1876. Nr. 1,

7) l. c, 
macht. Einen solchen Fall erhielt Leukart ${ }^{1}$ ) von Krïger in Braunschweig. Moniez ist geneigt, denselben als durch Entstehung zweier Proliferationspunkte neben einander aufzufassen und will ihn den Doppelmisbildungen zuzählen.

Als Teniae fenestratae hat man Tierstöcke bezeichnet, deren Glieder in grösserer oder geringerer Anzahl durchlöchert sind. Häufig sind die Öffnungen so gross, dass das ganze Mittelfeld fehlt und der Bandwurm einer Strickleiter nicht unähnlich ist. Beobachtungen dieser Art liegeni von Masars de Cazeles $^{2}$ ), Göze ${ }^{3}$ ), Colin ${ }^{4}$ ), Bremser ${ }^{5}$ ) und Leuckart ${ }^{6}$ ) vor. Platzen des Uterus scheint die wahrscheinlichste Ursache der Durchlöcherung zu sein. Die Erklärung, dass es sich um Verdauungserscheinungen handle, dïrfte nicht ausreichen, denn das ganze Aussehen des Wurms und die Glätte der WVundränder stehen damit in Widerspruch.

Die seltenste Art der Misbildung bei Taenien und auch wohl die an wenigsten beschriebene ist die Doppelmisbildung.

Bei dieser Bildungsanomalie giebt es nun Abstufungen zwischen zwei gleieherweise ansgebildeten und an einer Kante verwaehsenen Bandwürmern und einem Bandwurme, der auf der Fläehe seiner sämtliehen Glieder einen Longitudinalwulst aufzuweisen hat, der den Rest des verkïmmerten zweiten Bandwurms darstellt. Je naeh der geringeren oder grösseren Entwieklung dieses zweiten Bandwurms ist aneh die Zahl der Sangnäpfe am Seolex eine versehiedene, indem sie zwisehen 5, 5 und einer sehwachen Andentung eines 6. und 6 voll entwiekelten Sangnäpfen variirt. Die Vierzahl, in der sieh die Sangnäpfe der normalen Täniaden wiederholen, ist auf den Umstand zurüekgeführt, dass Baneh- und Riickenfläehe der Cestoden eine verhältnismässig nur geringe Differenzirung besitzen und besonders im Kopf kaum irgendwie von einander abweiehen. Es sind das Ver-

1) L. Parasiten des Menschen, p. 572.

9) M. d. C., Roux. Journ. T. XXIX., p. 26.

3) G., Eingeweidewürmer.

4) C., Gaz. des hôp. 1876. Nr. 1.

5) B., Lebende Würmer im lebenden Menschen. S. 94.

6) L., Parasiten des Menschen. S. 579. 
hältnisse, welehe sonst bei den Bilateraltieren nur selten in so ausgesprochener Weise uns entgegentreten, wohl aber das eharakteristisehe Merkmal der Radiärtiere bilden. Da ist es nun interessant, wenn wir sehen, dass die Köpfe der Täniaden gelegentlieh einer Misbildung unterliegen, der wir anch bei den vierstrahligen Radiärtieren nicht selten begegnen, einer Vermehrung nämlich der Radien auf 6 . Die so gebildeten Köpfe zeigen bei einer vergrösserten Hakenzahl 6 Sangnäpfe und 6 Längsgefässe anstatt der sonst gewöhnlichen 4. Dieselben sind wie im Normalzustande einander angenähert, sodass deren 3 Paare vorhanden sind. Gleichzeitig hat auch die Grösse des Kopfes zugenommen. Doch nicht nur der Kopf, auch der gegliederte Wurmkörper nimmt, wie sehon erwähnt, an dieser ungewöhnlichen Beschaffenheit Anteil. Hiermit im engen Zusammenhang stehen die prismatischen oder dreikantigen Ketten, die an sechsstrahligen Köpfen sich bilden.

Die ersten Nachrichten dariiber verdanken wir Bremser. Der Wurm bestand, wie Bremser ${ }^{1}$ ) sagt, aus zwei Ketten, die mittelst des einen Seitenrandes unter einem spitzen Winkel in ganzer Länge zusammen verwachsen sind und von annährungsweise gleicher Entwicklung waren. Der gemeinsehaftliehe Seitenrand sprang kantenartig vor und trug die Gesehleehtsöffnungen. Nur hier und da war noeh am freien Rande ein Porus genitalis vorhanden. Eine ähnliche Monstrosität hat Levacher ${ }^{2}$ ) beobachtet, nur dass hier die Art des Zusammenhangs insofern abweichend war, als die eine Kette der Fläehe der anderen in der Mittellinie aufsass. Die sonst bekannt gewordenen Fälle, die Cobbold'sche ${ }^{3}$ ) Taenia lophosoma und der Fall von Cullingworth ${ }^{4}$ sehliessen sich eng an die Verhältnisse des Bremer'schen Wurmes an. Aus dem pathologischen Institute zu Kiel sind zwei Dissertationen erschienen, die anch Taenienmisbildungen behandeln und zwar von Gustav Bork "Über die Misbildungen bei Taenien" (1891) und von Bernhardt Kürhel "Eine Drillingsmisbildung der Taenia sa-

1) B., Lebende Würmer im lebenden Menschen, p. 107.

2) L., Cpt. rend. T. XIII, p. 661 .

3) C., Transact. of the path. Soc. XV1I p. 438.

4) C., Medic. Tịmes and Gaz, 1873. 
ginata (1892). Erstere Dissertation hat eine von Herrn Geh. Medicinalrat Professor Dr. Heller gezeichnete Tafel, die unter anderen anch dic Abbildung einer Doppelmisbildung giebt, die ähnliche Verhältnisse zeigt, wie wir sic bei dem von mir weiterhim beschriebenen Wurm finden. Auch die von Kü̈chenmeister ${ }^{1}$ ) beschriebene „Taenie rom Cap der guten Hoffnnug" zeigt den gleichen Zusammenhang der beiden Ketten, nur dass die eine derselben eine sehr viel geringere Flächenentwicklung besitzt als die andere nurl kaum melır als einen Längswulst darstellt.

Hieran möchte ich den mir von Herru Geh. Medicinalrat Professor Dr. Heller güitigst iiberlassenen Fall von Doppelmisbildang amreihen.

Es ist auch hier eine Taenia saginata, die diese Misbildung aufzuweisen hat, und zwar ein wohlentwickeltes Exemplar von ca. $8 \mathrm{~m}$ Länge. Der Kopf, der $2 \frac{1}{2} \mathrm{~mm}$ im Durchmesser misst,

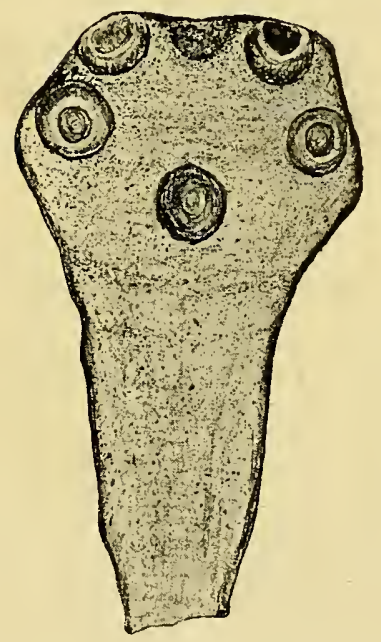

besitzt う̇ vollentwickelte und einen verkïmnerten Sangnapf. Der Hals ist kurz, denn schon wenige Millimeter hinter dem Kopfe treten bereits fuir das blosse Ange kenntliche Andentungen der

1) K., Über die im und am Menschen vorkommenden Parasiten. 2. Af. 
Glierlerung auf. Die ersten Proglottiden sind $2 \mathrm{~mm}$ breit nnd $1 \mathrm{~mm}$ lang, während die roll ansgewachsenen reifen Glieder in ersehlafftem Zustande eine Länge von $2 \mathrm{~cm}$ und eine Breite von $1 / 2$ em haben. Die Gesamtzahl der Bandwurmglieder mag ea. 2000 betragen. Wemn sich die Doppelmisbildung am Kopfe durch Vergrössermug desselben nud Vermehrung seiner Sangnäpfe kund thut, so tritt sie uns an den Proglottiden als seitlicher Längswulst entgegen. Wir haben also nieht zwei gleicherweise ansgebildete Bandwiirmer vor uns, sondern der eine ausgewachsene Bandwurm trägt ein verkümmertes Individum an seiner Seitenfläche. Während an mittleren Gliedern die Breite des Hanptwurmes $9 \mathrm{~mm}$ ist, beträgt die Höhe des Wulstes, also die Breite des verkïmmerten Wurmes, nur 2 mm. Dass aber dieser Läng'swulst trotz seiner geringen Hölıe als das Aequiralent eines Tierkörpers zu betrachten ist, geht nicht blos darans hervor, dass derselbe an der Gliedlerming der Kette teilnimmt, sondern weiter und hestimmter noch darans, dass er im Wesentlichen den gleichen Ban hat wie der Hanptkörper. An feinen Quersehnitten erkennt man an ihm die charakteristische Mittel- und Rindensehicht und sieht beide continuirlich in die entsprechenden Sehichten des Hauptkörpers iibergehen.

Sowohl an der beiden Körpern gemeinschaftlichen Kante als an den freien Ründern des breiteren Flïgels und des Wulstes, wenn auch an letzteren sehvach entwickelt, verlüuft ein Längsgefäss. Die Geschlechtsöffıungen sind unregelmässig wechselnd bald auf der gemeinschaftlichen Kante, bald an der Kante des breiteren Fliigels, jedoch hier in geringer Anzahl. An einer Stelle hat der Irurm sogar an 19 Gliedern hintereinander den Porus genitalis an der beiden Fliigeln gemeinsehaftliehen Kante. Hieraus kann man sich vielleicht erklären, dass fast alle Beohachter, denen nur Bruchstïcke ron solchen Würmern vorlagen, das Vorkommen von Gesehlechtsöffunngen am freien Flïgel gänzlich lengnen. Küichenmeister ${ }^{1}$ ) ist der einzige, dessen Beobachtungen mit den Verhältnissen am vorliegenden Wurme ïbereinstimmen. Am Wulste waren Gesehlechtsöffnungen nicht nachweishar.

1) K., Über die in und am Menschen vorkommenden Parasiten. 
Der Hauptstamm des Uterus verläuft da, wo die beiden Flïgel mit der Kante zusammenfliessen, an einer Stelle also, welehe wir als die morphologische Achse dieses Wurmes zu betrachten haben. Er hat inı allgemeinen das gewöhnliche Verhalten, nur sind die Verzweigungen weniger stark entwickelt. Der Längswulst bekommt die wenigsten und kürzesten Äste. Eier sind verhältnismässig wenig vorhanden und ein grosser Teil derselben zeigt auch noch pathologische Veränderungen.

Inbetreff der Verbindungsweise ist noch hervorzuheben, dass die Mittelebene des Wulstes mit dem Hauptkörper einen Winkel ven etwa $30^{\circ}$ bildet.

Eine sonst nicht seltene Misbildung, nämlich das Auftreten seitlich anhängender Glieder, ist an unserem Wurm besonders darum von Interesse, weil diese Glieder, zwei an der Zahl, nicht an der Doppelmisbildung teilnelmen. Wenn Moniez' Erklärung für die Entstehung anhängender Glieder richtig ist, dass nämlich in dem Falle an einer Proglottide zwei Proliferationspunkte nebeneinander liegen, so muss man diese Abweichung von der Doppelmisbildung dahin zu erklären suchen, dass der Proliferationspunkt des anhängenden Gliedes urspruinglich nur zum Hauptwur'm gehört.

Aus der Beschreibung dieser Taenie, die der' Küchenmeister'schen in vielen Stiicken gleicht, sehen wir also, dass der Hauptwurm hier noch eine gervisse Selbstständigkeit behalten hat, während bei den dreikantigen Würmern, wo beide Tiere gleicherweise ausgebildet sind, sich die Hauptorgane in der gemeinschaftlichen Kante zusammendrängen und auch nur an dieser Geschlechtsöffnungen zu finden sind. Auffallend und wohl einer besonderen Untersuchung wert ist bei unserer Misbildung das Verlalten der Eier, die erstigmal in geringer Anzahl vorhanden sind und ausserdem pathologische Veränderungen zeigen, so dass man zu der Vermutung kommen könnte, dass solche MLonstra steril wären. 
Zum Schlusse ist es mir eine angenehme Pflicht, Herrn Geh. Medicinalrat Professor Dr. Heller für die freundliche Überweisung dieser Arbeit, sowie für die guitige Überlassung des Materials meimen verbindlichsten Dank auszusprechen. 


\section{Lebenslauf.}

Geboren wurde ich, Hajo Jelden, erangelischer Confession, als Sohn des Pastors Heilo Jelden, am 13. Februar 1873 zu Holtland in Ostfriesland. Ich besuchte das Gymnasium zn Norden, das ich Ostern 1895 mit dem Zengnis der Reife rerliess, und widmete mich dann dem Studium der Medicin auf den Universitäten Tüibingen, Nünchen, Berlin. Kiel. Die ärztliche Torpriifung bestand ich am 5. Mär\% 1897 zu Tiibingen, beendete das medicinische Staatsexamen am 9. März 1900 hierselbst und unterzog mich dem Examen rigorosum am 12. März desselben .Jahres ebenfalls zu Kiel. Meiner halbjährigen Dienstzeit mit der Waffe genïgte ich rom 1. Oktober 1897 l,is 1. April 1898 bei dem Königl. Bayerischen Leibregiment in Minchen. 







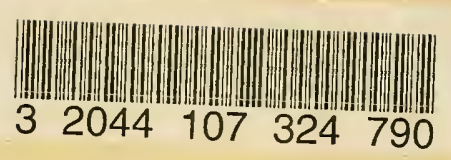


